Supporting Information for

\title{
Reusable Surface-enhanced Raman Spectroscopy \\ Membranes and Textiles via Template-assisted Self- assembly and Micro/nanoimprinting
}

Aditya Garg, Wonil Nam, Wei Zhou*

Department of Electrical and Computer Engineering, Virginia Tech, Blacksburg, Virginia 24061, United States

*Email:wzh@,vt.edu 

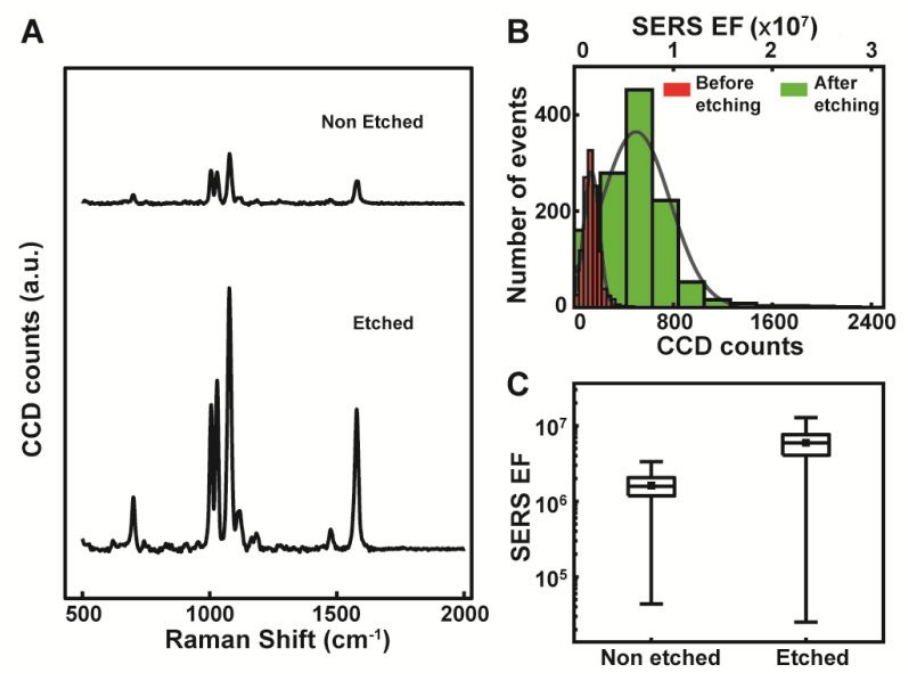

Figure S1. A) The measured Raman spectra for BZT molecules assembled on the surface of Au NP aggregates before RIE, and after 1 minute RIE under $785 \mathrm{~nm}$ laser excitation obtained with a 100x objective lens. B) Histogram of Raman signal intensities and corresponding EFs $\left(1077 \mathrm{~cm}^{-1}\right)$ before RIE and after 1-minute RIE treatment. C) Boxplots of SERS EF before RIE and after 1-minute RIE treatment. The five different bars from top to bottom represent the max, $75 \%$, median, $25 \%$, and min values of SERS EFs, respectively. 


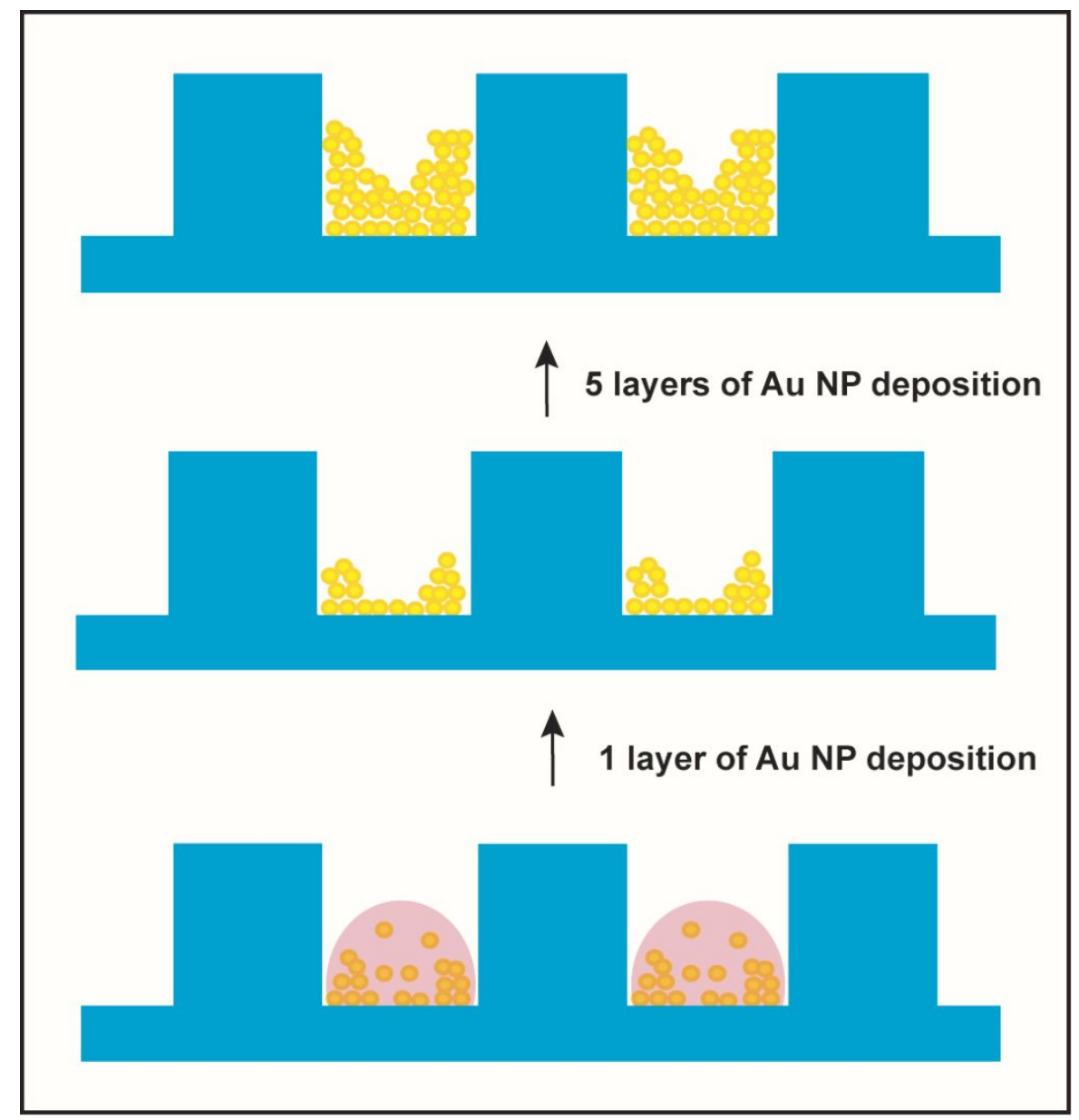

Figure S2. Schematic depicting the proposed mechanism for the confinement of Au NPs within the microwell array. 

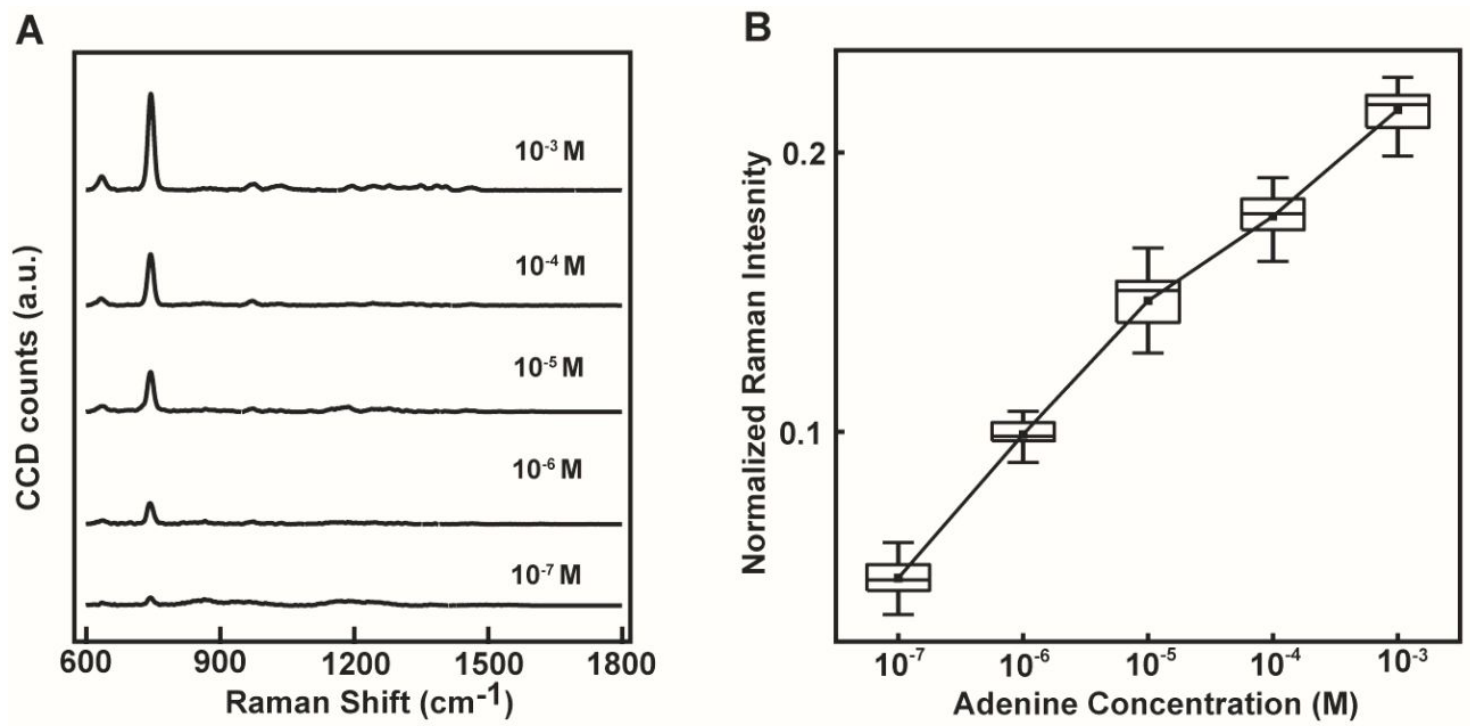

Figure S3. SERS detection of adenine. A) Measured Raman spectra under $785 \mathrm{~nm}$ excitation of different concentrations of adenine solution on the micropatch array of $\mathrm{Au}$ NP aggregates and B) the corresponding boxplots of normalized Raman intensity $\left(732 \mathrm{~cm}^{-}\right.$ 1) for $10^{-7}, 10^{-6}, 10^{-5}, 10^{-4}$, and $10^{-3} \mathrm{M}$ adenine. The five different bars from top to bottom represent the max, $75 \%$, median, $25 \%$, and min values of normalized Raman intensity respectively. 